\title{
Fuzzy Delphi and hybrid AH-MATEL integration for monitoring of paint utilization
}

\author{
Kumar, A. ${ }^{a}{ }^{,}$, Mussada, E.K. ${ }^{a}$, Ashif, M. ${ }^{b}$, Tyagi, D. $^{b}$, Srivastava, A.K. ${ }^{b}$ \\ ${ }^{a}$ BML Munjal University, Gurgaon, India \\ ${ }^{b}$ MBA-Hero Lead Students, BML Munjal University, Gurgaon, India
}

\begin{abstract}
A B S T R A C T
This study investigates the unattended aspects of paint utilization selection criteria in industries. In today competitive business environment almost all companies focus towards sustainable manufacturing. The utilization evaluation and selection criteria for paint and its consumption reduction is the top priority for industry. Especially in automotive industries, paint shop stands as a centre for hazardous waste due to wastage of paint and thinner during the painting process. This research work focuses on optimizing consumption of paint by finding most important criteria affecting paint consumption and optimizing the same to achieve maximum paint yield. The study uses the routes of Delphi technique in a fuzzy environment to find out the most important criteria for paint utilization selection, so that maximize utilization and minimize consumption reduction of paint has been achieved. An integrated approach of AHP and DEMATEL methods has been implemented to prioritize the criteria and to familiarize the relationship within criteria. The outcomes of the study substantiate and prove that this study is the best way to select particular paint utilization selection criteria for the paint shop and also to anticipate the optimal level of paint utilization.
\end{abstract}

\author{
ARTICLE INFO \\ Keywords: \\ Automotive industry \\ Paint shop \\ Optimization \\ Paint consumption and utilization \\ AHP \\ DEMATEL \\ *Corresponding author: \\ anilror@gmail.com \\ (Kumar, A.) \\ Article history: \\ Received 20 July 2016 \\ Revised 13 February 2017 \\ Accepted 15 February 2017
}

(C) 2017 PEI, University of Maribor. All rights reserved.

\section{References}

[1] Büyüközkan, G., Çifçi, G. (2012). A combined fuzzy AHP and fuzzy TOPSIS based strategic analysis of electronic service quality in healthcare industry, Expert Systems with Applications, Vol. 39, No. 3, 2341-2354, doi: 10.1016/j.eswa.2011.08.061.

[2] Chang, P.-T., Huang, L.-C., Lin, H.-J. (2000). The fuzzy Delphi method via fuzzy statistics and membership function fitting and an application to the human resources, Fuzzy Sets and Systems, Vol. 112, No. 3, 511-520, doi: 10.1016/S0165-0114(98)00067-0.

[3] Chen, H.H., Kang, H.Y., Xing, X., Lee, A.H.I., Tong, Y. (2008). Developing new products with knowledge management methods and process development management in a network, Computers in Industry, Vol. 59, No. 2-3, 242253, doi: 10.1016/j.compind.2007.06.020.

[4] Dalalah, D., Hayajneh, M., Batieha, F. (2011). A fuzzy multi-criteria decision making model for supplier selection, Expert Systems with Applications, Vol. 38, No. 7, 8384-8391, doi: 10.1016/i.eswa.2011.01.031.

[5] Dalkey, N., Helmer, O. (1963). An experimental application of the Delphi method to the use of experts, Management Science, Vol. 9, No. 3, 458-467, doi: $10.1287 / \mathrm{mnsc}$.9.3.458.

[6] Ghorabaee, M.K. (2016). Developing an MCDM method for robot selection with interval type-2 fuzzy sets, Robotics and Computer-Integrated Manufacturing, Vol. 37, 221-232, doi: 10.1016/j.rcim.2015.04.007.

[7] Ho, W.-R.J., Tsai, C.-L., Tzeng, G.-H., Fang, S.-K. (2011). Combined DEMATEL technique with a novel MCDM model for exploring portfolio selection based on CAPM, Expert Systems with Applications, Vol. 38, No .1, 16-25, doi: 10.1016/j.eswa.2010.05.058. 
[8] Hung, Y.-H., Huang, T.-L., Hsieh, J.-C., Tsuei, H.-J., Cheng, C.-C., Tzeng, G.-H. (2012). Online reputation management for improving marketing by using a hybrid MCDM model, Knowledge-Based Systems, Vol. 35, 87-93, doi: 10.1016/j.knosys.2012.03.004.

[9] Ishikawa, A., Amagasa, M., Shiga, T., Tomizawa, G., Tatsuta, R., Mieno, H. (1993). The max-min Delphi method and fuzzy Delphi method via fuzzy integration, Fuzzy Sets and Systems, Vol. 55, No. 3, 241-253, doi: 10.1016/0165-0114(93)90251-C.

[10] Lin, Y.-H., Tseng, M.-L. (2016). Assessing the competitive priorities within sustainable supply chain management under uncertainty, Journal of Cleaner Production, Vol. 112, Part 3, 2133-2144, doi: 10.1016/j.jclepro.2014. $\underline{07.012 .}$

[11] Malik, M.M., Abdallah, S., Hussain, M. (2016). Assessing supplier environmental performance: Applying analytical hierarchical process in the United Arab Emirates healthcare chain, Renewable and Sustainable Energy Reviews, Vol. 55, 1313-1321, doi: 10.1016/j.rser.2015.05.004.

[13] Tadić, S., Zečević, S., Krstić, M. (2014). A novel hybrid MCDM model based on fuzzy DEMATEL, fuzzy ANP and fuzzy VIKOR for city logistics concept selection, Expert Systems with Applications, Vol. 41, No. 18, 8112-8128, doi: 10.1016/i.eswa.2014.07.021.

[14] van Zolingen, S.J., Klaassen, C.A. (2003). Selection processes in a Delphi study about key qualifications in senior secondary vocational education, Technological Forecasting and Social Change, Vol. 70, No. 4, 317-340, doi: 10.1016/S0040-1625(02)00202-0.

[15] Rao, R.V., Rai, D.P., Ramkumar, J., Balic, J. (2016). A new multi-objective Jaya algorithm for optimization of modern machining processes, Advances in Production Engineering \& Management, Vol. 11, No. 4, 271-286, doi: 10.14743/apem2016.4.226.

[16] Kumar, A., Dash, M.K. (2016). Using DEMATEL to construct influential network relation map of consumer decision-making in e-marketplace, International Journal of Business Information Systems, Vol. 21, No. 1, 48-72, doi: 10.1504/IJBIS.2016.073380.

[17] Wu, W.-W. (2008). Choosing knowledge management strategies by using a combined ANP and DEMATEL approach, Expert Systems with Applications, Vol. 35, No. 3, 828-835, doi: 10.1016/i.eswa.2007.07.025.

[18] Wu, H.-H., Tsai, Y.-N. (2012). An integrated approach of AHP and DEMATEL methods in evaluating the criteria of auto spare parts industry, International Journal of Systems Science, Vol. 43, No. 11, 2114-2124, doi: $10.1080 / 00207721.2011 .564674$.

[19] Jafari-Moghadam, S., Zali, M., Sanaeepour, H. (2017). Tourism entrepreneurship policy: A hybrid MCDM model combining DEMATEL and ANP (DANP), Decision Science Letters, Vol. 6, No. 3, 233-250, doi: 10.5267/ j.dsl.2016.12.006.

[20] Saaty, T.L. (2008). Decision making with the analytic hierarchy process, International Journal of Services Sciences, Vol. 1, No. 1, 83-98.

[21] Kumar, A., Dash, M.K. (2014). Criteria exploration and multi-criteria assessment method (AHP) of multigenerational consumer in electronic commerce, International Journal of Business Excellence, Vol. 7, No. 2, 213 236, doi: 10.1504/IJBEX.2014.059549. 


\section{APEM}

\title{
Integracija metode mehki Delphi in hibridne metode AH-METAL za spremljanje porabe barve
}

\author{
Kumar, A. ${ }^{a}{ }^{,}{ }^{,}$, Mussada, E.K. ${ }^{a}$, Ashif, M. ${ }^{b}$, Tyagi, $^{\text {D. }}{ }^{b}$, Srivastava, A.K. ${ }^{b}$ \\ ${ }^{a}$ BML Munjal University, Gurgaon, India \\ ${ }^{\mathrm{b}}$ MBA-Hero Lead Students, BML Munjal University, Gurgaon, India
}

\section{POVZETEK}

Ta študija proučuje merila za izbiro barve v industriji. V današnjem tekmovalnem okolju se večina podjetij osredotoča na trajnostno proizvodnjo. Vrednotenje porabe in merila za izbiro barve ter zmanjšanje njene porabe so najvišja prioriteta industrije. Predvsem v avtomobilski industriji so lakirnice zaradi odpadnih barv in razredčil središče nastajanja nevarnih odpadkov. Ta raziskava se osredotoča na optimizacijo porabe barv z določitvijo najpomembnejših parametrov, ki vplivajo na njeno porabo in z optimizacijo le-teh, $\mathrm{z}$ namenom doseganja najvišje stopnje izkoriščenosti barv. Študija z Delphi tehniko v mehkem okolju poišče najpomembnejša merila za izbiro barve, ki zagotavljajo najvišjo stopnjo izkoriščenosti barv in zmanjšajo njen odpad. Za določitev prioritete meril in za vzpostavitev povezav med njimi je uporabljena integrirana AHP in DEMATEL metoda. Rezultati raziskave utemeljijo in dokažejo, da je takšna študija najboljša pot za določitev meril za izbiro barve za lakirnico ter da je primerna tudi za napoved najprimernejše ravni uporabe barve.

\section{PODATKI O ČLANKU}

Ključne besede:

Avtomobilska industrija

Lakirnica

Optimizacija

Poraba in uporaba barve

AHP

DEMATEL

*Kontaktna oseba: anilror@gmail.com (Kumar, A.)

Zgodovina članka: Prejet 20. julija 2016

Popravljen 13. februarja 2017

Sprejet 15. februarja 2017 